\title{
Determination of Flux Density of the Solar Radio Burst Event by Using Log Periodic Dipole Antenna (LPDA)
}

\author{
Z. S. Hamidi ${ }^{1, *}$, N. N. M. Shariff ${ }^{2, * *}$ \\ 1 MARA University of Technology, 40450, Shah Alam, Selangor, Malaysia \\ ${ }^{2}$ Department of Science and Technology Studies, University of Malaya, \\ 50603, Kuala Lumpur, Malaysia \\ *,**E-mail address: zetysh@salam.uitm.edu.my , nur.nafhatun@um.edu.my
}

\begin{abstract}
In this article, an evaluation of the flux density of the solar radio burst is presented. A rod aluminium's type as a conductor with nineteenth (19) elements of different sizes is being prepared to construct a log periodic dipole antenna (LPDA) from 45-870 MHz. The performance was carried out at the National Space Agency (PAN), Sg. Lang, Banting Selangor by connecting to the Compound Low Cost Low Frequency Spectroscopy Transportable Observatory (CALLISTO) spectrometer. The input impedance, $\mathrm{R}_{0}=50 \mathrm{ohm}$ is chosen for this LPDA antenna. From the analysis, the gain of the antenna is $9.3 \mathrm{~dB}$. This antenna potentially captures a signal that covers about $0.08 \mathrm{~m}^{2}$ area of the Sun.The temperature of the burst that detected at the feedpoint of the antenna is $32 \mathrm{~K}$. However, the signal becomes decrease to $28.75 \mathrm{~K}$ while by CALLISTO spectrometer as a receiver. It was also found that the isotropic source spectral power is $1576 \mathrm{~W} / \mathrm{Hz}$. Since the burst level above the background sky is $0.41 \mathrm{~dB}$, the flux density of the burst is $5.5 \times 10^{-21} \mathrm{~W} / \mathrm{m}^{2} / \mathrm{Hz}$. Based on the results, we conclude that this antenna is suitable for to observe the Sun activities at low frequency region.
\end{abstract}

Keywords: Sun; CALLISTO; Log Periodic Dipole Antenna (LPDA); power flux density; solar radio burst

\section{INTRODUCTION}

The Log-Periodic Dipole Antenna (LPDA), first built in 1958 by Du Hamel and Dwight Isbell, an undergraduate researcher in the ECE Antenna Laboratory and the prepared by Carrel [1,2]. Since that, this type of antenna has been used widely and become as one of the essential parts in the recent explosion in information technology and wireless communications. In principle, this application lies the study of electromagnetic energy in an unbounded medium or from space which can be detected with a special arrangement of conductors. Simple design and effective detector are the most factor why this type is still relevant. Moreover, this antenna is also easy to construct and suitable to monitor the solar activities. The radiation characteristics vary considerably below the lower frequency limit of the antenna. 
In solar radio astronomy, LPDA also been used to monitor solar activities of the sun based on the flux density of the burst. During the explosion, these active regions relatively have strong magnetic fields with denser and hotter compared with their surroundings and enhanced radio burst typically from 10-100 minutes. This flux density is a main parameter to study the mechanisms of radio emission at specific wavelengths associated with physical condition in the source environment. The antenna can be used independently or by connecting to the dish of radio telescope with a tracking system. This detection is formed as a solar radio burst type. Generally, there are five (5) solar radio burst designed as 1-V and each type is associated with different phenomena [3]. Solar radio burst type I is associated with a solar storm and an indicator of the initial eruption of the active region of the sun while type II is normally associated with Coronal Mass Ejections (CMEs) events. This phenomena is one of the interesting phenomena that has been monitored since 1970s. Type II can be divided into two sub-types: (i) a hearing bones and (ii) harmonic structure. Type III and V occurred during solar flares. Meanwhile, type III is very dominant and could be observed frequently by singular, in groups, or storms compare with other burst. It can be identified as a drifting burst which is drifting from high to low frequencies. However type $\mathrm{V}$ is quiet rare and it has a smooth but short-lived continuum characteristics. The beginning of the new active region can be characterized by the type IV burst with a broad continuum and a fine structure [4].

There are some basic parameters that might affect the performance of the antenna. In order to get a better picture of the performance of LPDA, one of the methods that can be done is by determining the power flux density of the burst. This is important to distinguish the performance of LPDA during solar events occurred. There might be other factors that will affect the performance of LPDA such as interference from other nearest sources. The radiation pattern, polarization, operation of the frequency band, gain, input impedance and efficiency of an antenna which indicates the power or field strength radiated in any direction relative to that in the direction of maximum radiation also should be considered.

\section{METHODOLOGY}

There are some basic parameters that might affect the performance of the antenna. In order to get a better picture of the performance of LPDA, one of the methods that can be done is by determining the power flux density of the burst. This is important to distinguish the performance of LPDA during solar events occurred. There might be other factors that will affect the performance of LPDA such as interference from other nearest sources. The radiation pattern, polarization, operation of the frequency band, gain, input impedance and efficiency of an antenna which indicates the power or field strength radiated in any direction relative to that in the direction of maximum radiation also should be considered.

The LPDA is a coplanar linear array of unequal and unequally spaced parallel linear dipoles fed by a twisted balanced transmission line. It consists of small, closely spaced half-wave dipoles. These dipoles are connected to the source using a twin transmission line in such a way that the phase is reversed at each connection relative to the adjacent elements. Each dipole is effective over a narrow band of frequencies determined by its length.

We have constructed the antenna at the Radio Cosmology Laboratory Physics Department, University of Malaya. One should consider which the best material that practical is and be able to conduct a current. 


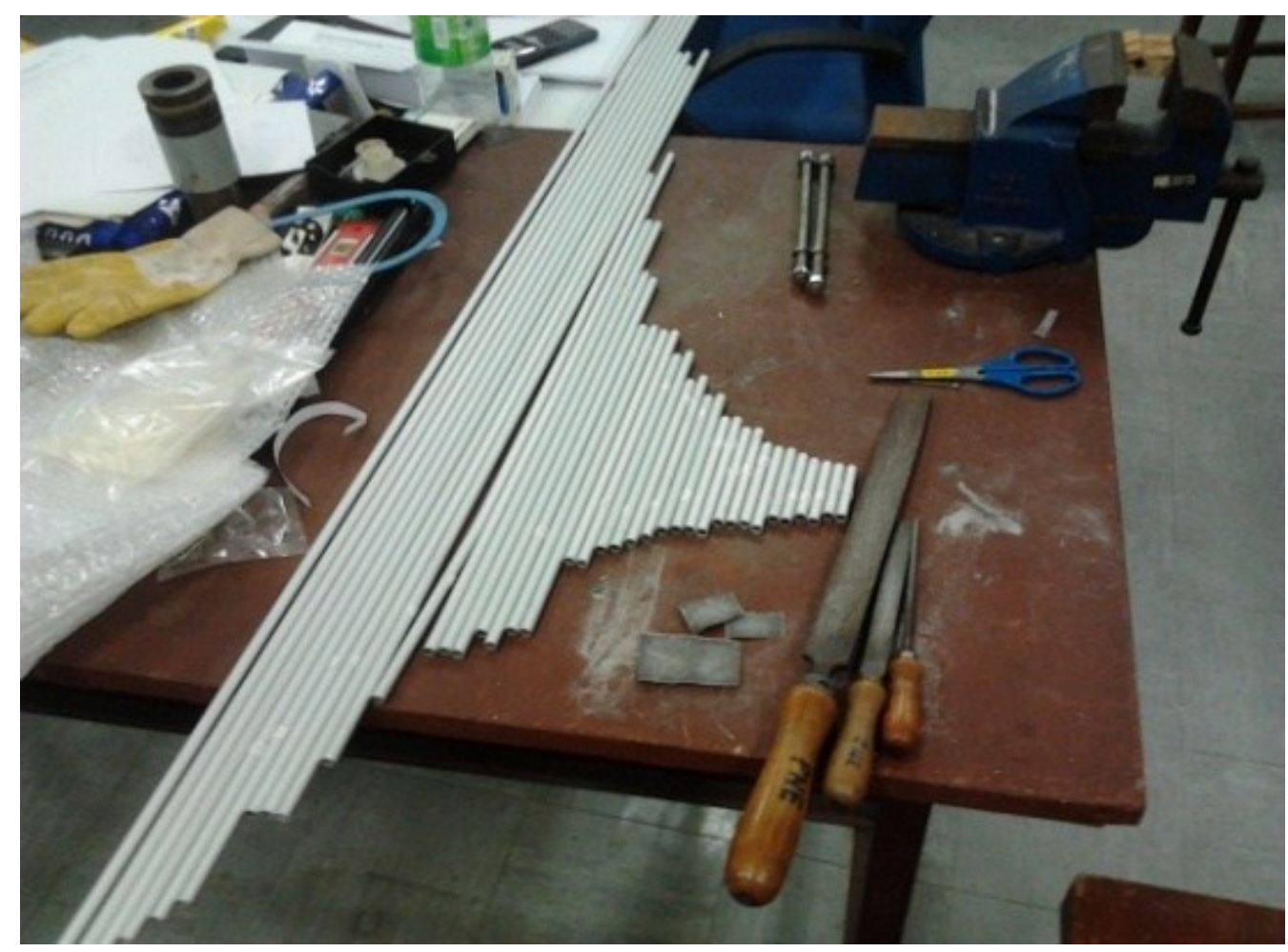

Figure 1. Preparation of construction of LPDA.

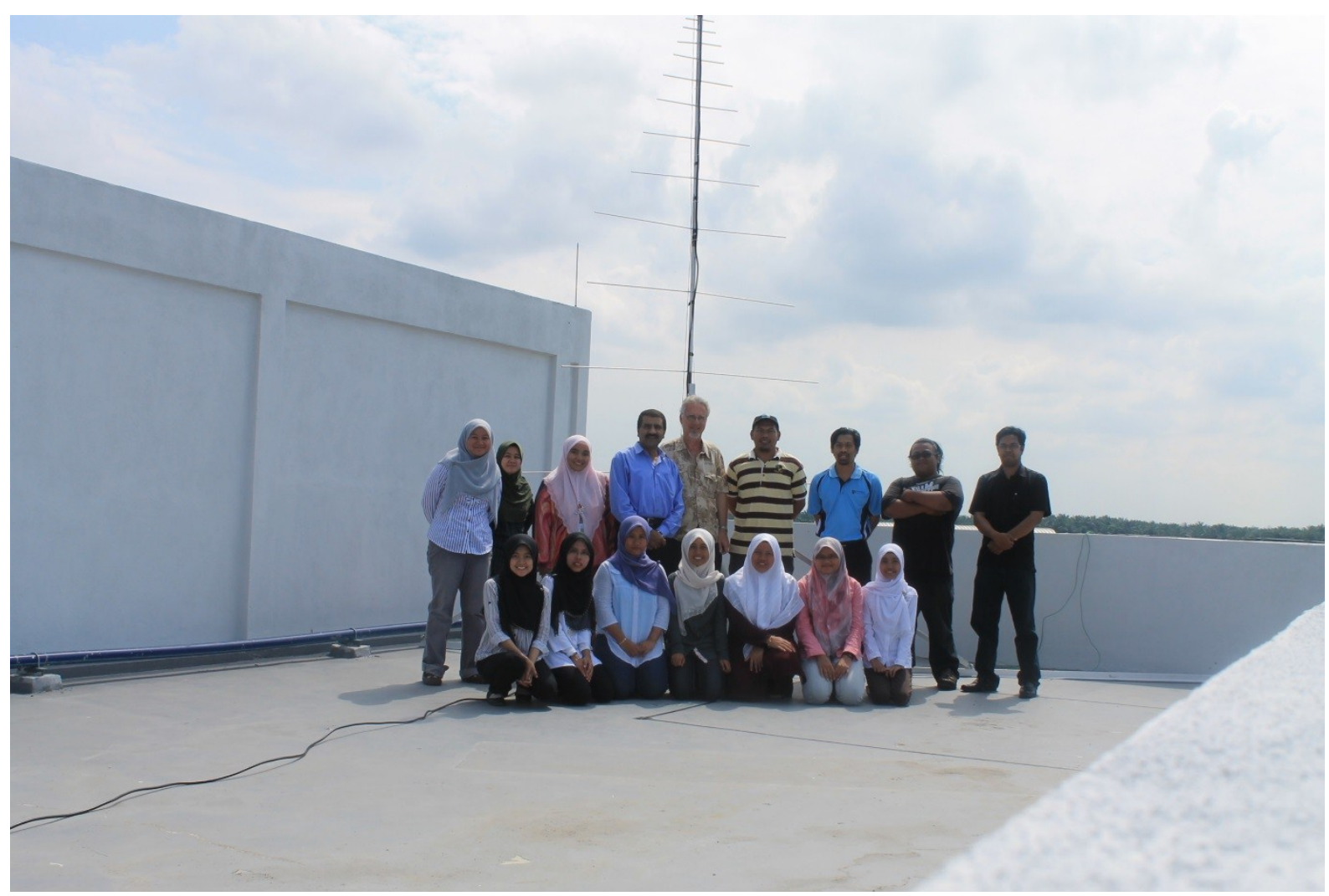

Figure 2. Assemblies of LPDA at National Space Centre, Banting, Malaysia. 
Here, an aluminium type is being chosen seems it can be used for outdoor purpose with PVC insulator between the booms. It is tough, and does not rust as it has a protective oxide coating which quickly forms on freshly exposed aluminium. Beside inexpensive, it is easy to handle as it is light in weight. We cut it in different sizes based on frequency. It should be noted that the dimension of the antenna is based on the range of the frequency that we decided to observe. The maximum size of the element is 1.11 meters and the minimum size is 0.02 meters. Figure. 1 shows the preparation of the element of the antenna.

In order to obtain more gain than with a single log-periodic array, two (2) arrays may be stacked. The diameter of these rods and tubes were chosen so as to incorporate the compactness as well as the ease of assembly for the antenna elements. Since it is dealing with transverse waves, all aerials are polarized. As this antenna is not automatically tracking yet, we decided to make sure that the directivity of an antenna is concentrated to the point of zenith direction. This is because the radiation of the Sun is maximum during this point and the LPDA is more stable in this direction. Figure. 2 shows the assembly of the LPDA at National Space Center Banting, Selangor Malaysia.

\section{RESULTS AND ANALYSIS}

The input impedance, $\mathrm{R}_{0}=50 \Omega$ is chosen for this LPD antenna [5]. For an efficient transfer of energy, the impedance of the antenna and the impedance of the transmission cable connecting them must be the same and matched.

An alternating current element occurred to be a basic source of radiation according to the specification of the dimension of LPDA. Based on theory, the performance of LPDA is a function of the antenna parameters $\tau$ and $\alpha$ [6]. The input impedance will increase toward the value of the unloaded feeder impedance as the value of $\tau$ is decreased. In particular, the input impedance depends on $\tau$ and $\alpha$. In this case, it can be considered as a broadband type with a wide band of $825 \mathrm{MHz}$ within 5.5 meters. As a consequence, the longest dipole in the array will be $3.96 \mathrm{~m}$ long [7]. It should be noted that the longer the boom the better the gain. However, due to the mechanical factors, it is not easy to construct a longer LPDA. The weight and dimension of the elements will make the antenna not become unstable. Unless, the material can be changed by using a copper, then it would be improved [8-10].

The chosen tau $(\tau)$ and sigma $(\sigma)$ give in the subtended angle of 3.43 degrees. As indicated in Figure 1, this LPDA antenna will have 19 elements. This antenna is connected CALLISTO (Compound Astronomical Low Cost Low Frequency Instrument Spectroscopy Transportable Observatory) spectrometer in the operation room. This international network solar radio spectrometer is designed by the Institute of Astrophysics, ETH Zurich, Switzerland under the International Space Weather Initiative (ISWI) project in order to monitor solar activities in radio region within 24 hours. This spectrometer e-C07 having a detector sensitivity of $25 \mathrm{mV} / \mathrm{dB}$ including control cables and radio frequency adapters was supplied by ETH Zurich. It consists a channel with $62.5 \mathrm{kHz}$ resolution, while the radiometric bandwidth is about $300 \mathrm{kHz}$. For each sample, it will take exactly $1.25 \mathrm{msec}$ per frequencypixel while the integration time is about $1 \mathrm{msec}$. With large instantaneous bandwidths and high spectral resolutions, these instruments will provide increased imaging sensitivity and enable detailed measurements of the dynamic solar burst. Up to date, there are 30 sites all over the world that used the same system.

This collaboration is an alternative project in order to support a new country that is interested to study solar astrophysics. 
The output from the transmitter is converted into electromagnetic energy by the antenna and radiated into space. The feed point for the array is seen to increase smoothly in dimension, being greater for each element than the previous element in the array. At the receiving antenna, the electromagnetic energy is collected and converted into electrical energy and fed to the receiver. Only a tiny fraction of power is reflected back to the spectrum analyzer indicating that most of the power has been radiated by the antenna. Details values of the dimensions of the design of the LPDA are illustrated in Table 1. Detection of solar burst type III has been observed on 9th March 2012 (about 3:32 UT till 5:00 UT) which is approximately occurring in 1 and half hour (88 minutes). We focused on the duration starting from the pre-stage of solar flare till the post stage of the flare. Associated with a type III burst is type $\mathrm{U}$ radio burst.

Table 1. The physical dimension of LPDA.

\begin{tabular}{|c|c|c|c|}
\hline $\begin{array}{c}\text { No of } \\
\text { elements }\end{array}$ & $\mathrm{L}(\mathrm{m})$ & $\mathrm{R}(\mathrm{m})$ & $\mathrm{D}(\mathrm{m})$ \\
\hline 1 & 3.96533329 & 5.551466963 & 1.110293326 \\
\hline 2 & 3.17226668 & 4.441173636 & 0.888234674 \\
\hline 3 & 2.53781338 & 3.552938962 & 0.710587750 \\
\hline 4 & 2.03025073 & 2.842351212 & 0.568470208 \\
\hline 5 & 1.62420061 & 2.273881003 & 0.454776173 \\
\hline 6 & 1.29936050 & 1.819104830 & 0.363820944 \\
\hline 7 & 1.03948842 & 1.455283885 & 0.291056759 \\
\hline 8 & 0.83159075 & 1.164227125 & $0 . .232845411$ \\
\hline 9 & 0.66527261 & 1.164227125 & 0.186276331 \\
\hline 10 & 0.53221809 & 0.745105382 & 0.149021067 \\
\hline 11 & 0.42577448 & 0.596084315 & 0.119216855 \\
\hline 12 & 0.34061959 & 0.476867459 & 0.095373486 \\
\hline 13 & 0.27249567 & 0.381493973 & 0.076298790 \\
\hline 14 & 0.21799654 & 0.305195182 & 0.061039032 \\
\hline 15 & 0.17439723 & 0.244156150 & 0.048831227 \\
\hline 16 & 0.13951779 & 0.195324922 & 0.039064982 \\
\hline 17 & 0.11161423 & 0.156259940 & 0.031251986 \\
\hline 18 & 0.08929139 & 0.125007954 & 0.025001589 \\
\hline 19 & 0.07143311 & 0.100006365 & 0.020001271 \\
\hline
\end{tabular}




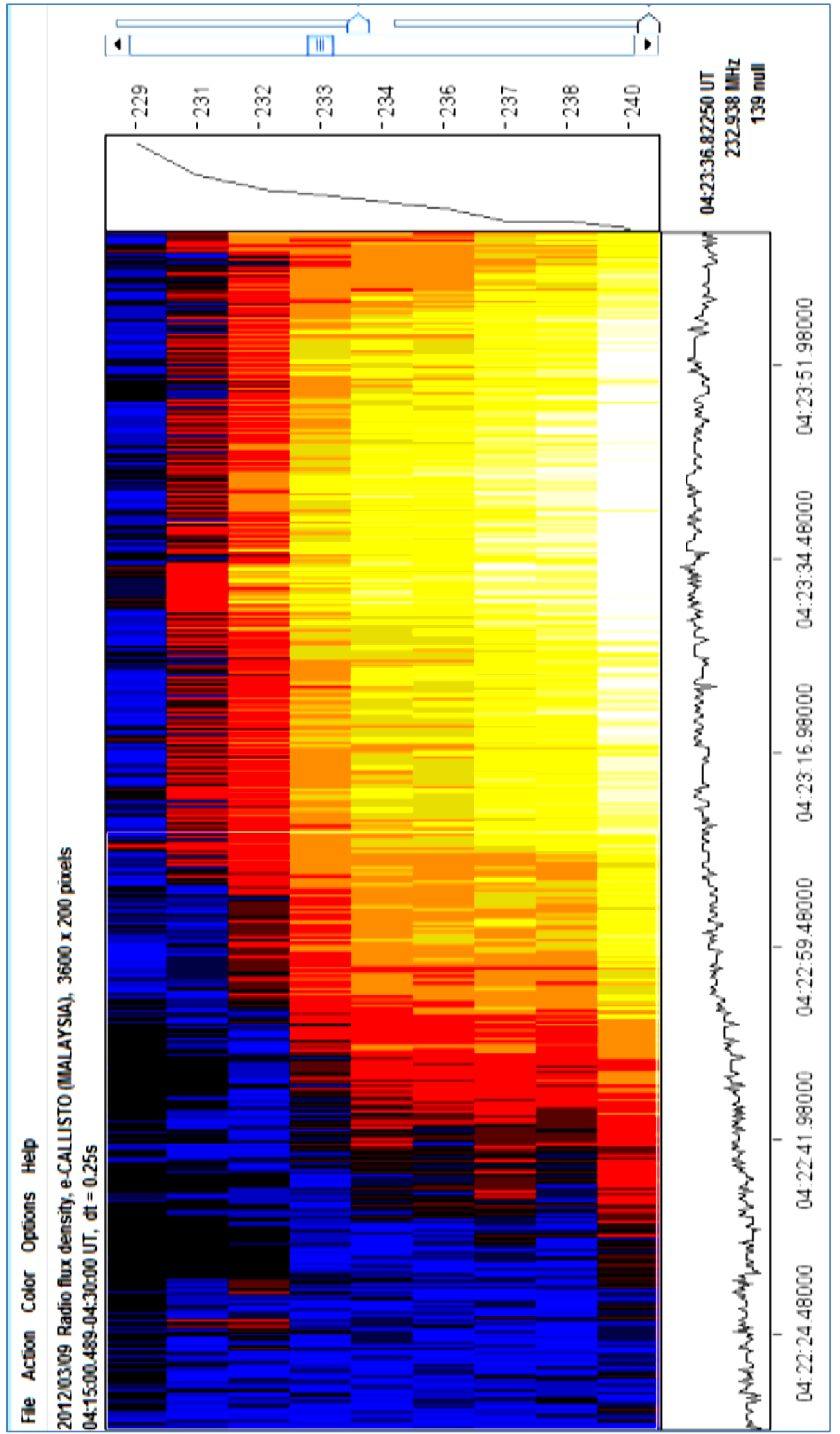

Figure 3. The cold (blue) and hot (yellow) section of solar radio burst. 
Once the LPDA is successfully constructed, we test the antenna by evaluating the power flux density of solar burst. Therefore, we chose one of a good data. Solar radio burst type III that have been detected during $9^{\text {th }}$ March 2012 associated with a large solar flare, type M6.3 has been chosen in this case. Based on calculation the temperature of the solar burst is 328.9 $\mathrm{K}$. It was found that the background sky temperature of the receiver of the antenna is 300.15 K. Figure 3 shows the solar burst (bright color) and sky background (dark color) from the observations.

In order to calculate the Signal to Noise Ratio (SNR), the Power Flux Density (PFD) will be measured. However, it is necessary to calculate the effective area of the antenna, $A_{\text {eff }}$ at the first stage. In this case, the symbol $\mathrm{G}$ represents gain and $\mathrm{G}(\mathrm{dB})$ is a gain in $\mathrm{dB}$ units. Since the solar burst data is at frequency, $\mathrm{f}=240 \mathrm{MHz}$, this value will be selected and $\mathrm{c}$ is the speed of light in $\mathrm{m} / \mathrm{s}$.

We are now in the process of testing the antenna during the observation of the Sun. In analysis process, we used a flux density and spectral power of Jovian and solar burst software to determine the other important parameters. One big challenge of this range is the population of different interference source that might affect the solar burst data. Yet it is still can be eliminated during the analysis process.Detailed analysis of LPDA and solar burst parameter is illustrated in Figure 4.

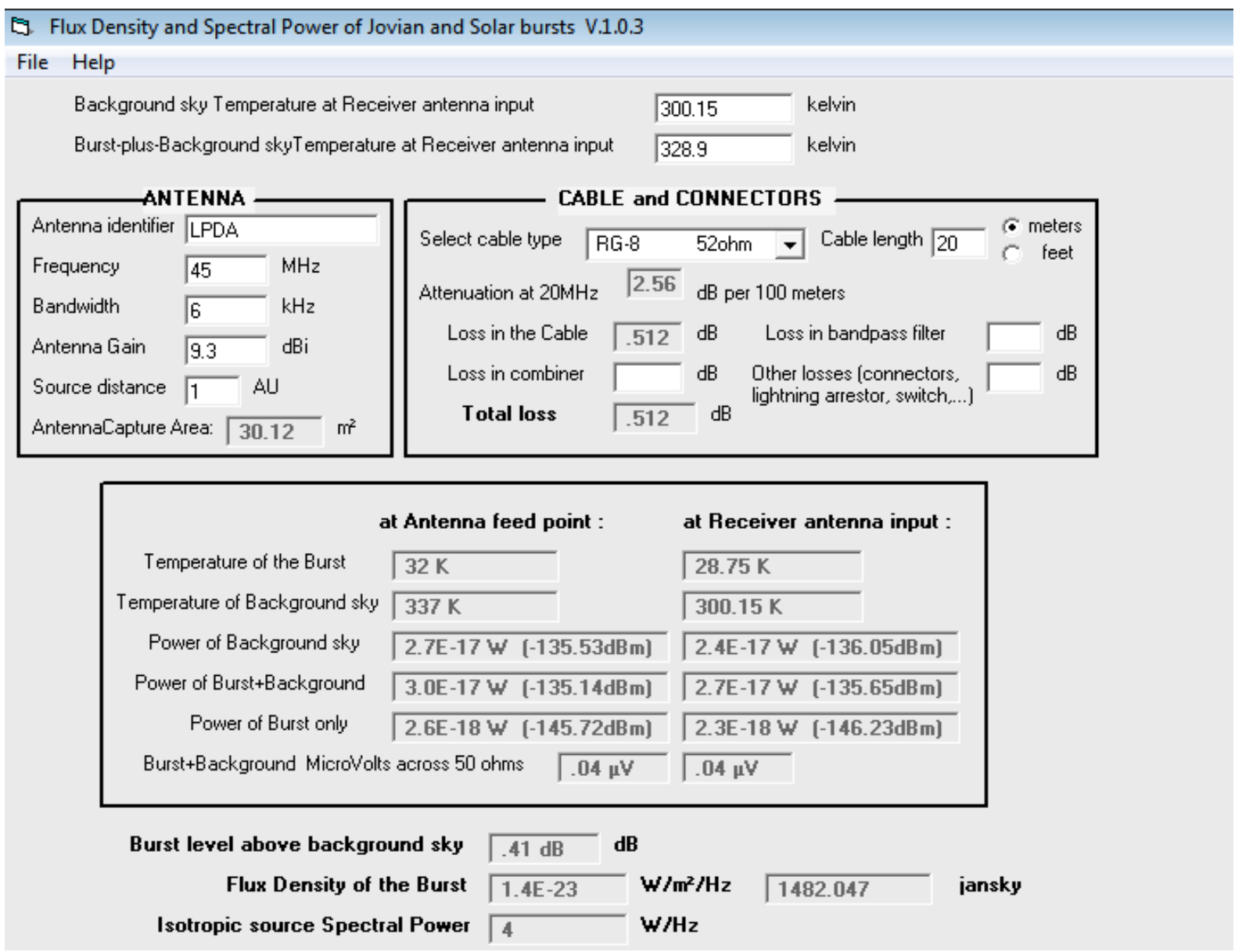

Figure 4. The flux density software of solar burst.

From the analysis process, it was found that the gain of the antenna is $9.3 \mathrm{~dB}$. This antenna potentially captures a signal that covers about $0.08 \mathrm{~m}^{2}$ area of the Sun. By using 20 
meters long of RG8 cable ( $52 \Omega$ ), there will be about $2.56 \mathrm{~dB}$ of attenuation for every 20 meters. On average, we can say that the signal will lose about $0.512 \mathrm{~dB}$ per 100 meters.

In addition, the temperature of the burst that detected at the feedpoint of the antenna is $32 \mathrm{~K}$. However, The signal becomes decrease to $28.75 \mathrm{~K}$ while by the CALLISTO spectrometer as a receiver. It was found that the isotropic source spectral power is 1576 $\mathrm{W} / \mathrm{Hz}$. Since the burst level above the background sky is $0.41 \mathrm{~dB}$, the flux density of the burst is $5.5 \times 10^{-21} \mathrm{~W} / \mathrm{m}^{2} / \mathrm{Hz}$.

\section{CONCLUSION}

While the main goal of wideband imaging is to obtain a high dynamic-range continuum solar burst profile, the reconstructed spectral structure can also be a useful astrophysical measurement. The flux density of the solar burst has been evaluated. Hence, this parameter is important seems the temperatures are different but the flux density, that is, the power landing on a square meter per hertz of bandwidth will be the same. Therefore, this is one way of calibration method that should be doing as a routine to make sure that the antenna is in a good performance. Our next task is to observe the radiation pattern of the signal of the LPDA. In conclusion, this antenna is suitable to observe the solar activities at low frequency region.

\section{ACKNOWLEDGEMENT}

This work was partially supported by the PPP UM PV071/2011B grants. Special thanks to C. Monstein from ETH Zurich, Switzerland who set up and gives us training on analyzing the data. Also to the National Space Agency and the National Space Centre for giving us a site to set up this project and support this project. Solar burst monitoring is a project of cooperation between the Institute of Astronomy, ETH Zurich, and FHNW Windisch, Switzerland, MARA University of Technology and University of Malaya. This paper also used NOAA Space Weather Prediction Centre (SWPC) for the sunspot, radio flux and solar flare data for comparison purpose. The research has made use of the National Space Centre Facility and a part of an initiative of the International Space Weather Initiative (ISWI) program.

\section{BIOGRAPHY}

Zety Sharizat Hamidi is currently a PhD candidate and study in Solar Astrophysics specifically in radio astrophysics at the University of Malaya. Involve a project under the International Space Weather Initiative (ISWI) and also a lecturer in School of Physics and Material Science, at MARA University of Technology, Shah Alam Selangor.

N. N. M. Shariff Her current research is communicating sustainability. She is looking forward for cross-field research i.e. solar astrophysics, light pollution measurement (mapping) and application of technology on sustainability.

\section{References}

[1] R. H. DuHamel, D. E. IsBell, Broadband Logarithmically Periodic Antenna Structures, IRE national convention record, 1957, pp. 119-128.

[2] R. L. Carrel, Analysis and Design of the Log-Periodic Dipole Antenna, Elec. Eng. Dept., University of Illinois, 1961.

[3] M. R. Kundu, Solar Radio Astronomy, John Wiley, 1965.

[4] J. P. Wild, Smerd S. F., Weiss A. A., Ann. Rev. Astron. Astrophysics 1 (1963) 291-366. 
[5] Z. S.Hamidi, et al., International Journal of Fundamental Physical Sciences 2 (2012) 4.

[6] C. E. Smith, Log Periodic Antenna Design Handbook, 1st ed., Ohio, 1966.

[7] Z. Hamidi, S. Chumiran, A. Mohamad, N. Shariff, Z. Ibrahim, N. Radzin, N. Hamzan, N. Anim, A. Alias, American Journal of Modern Physics 2 (2013) 4.

[8] Z. S. Hamidi, N. N. M. Shariff, International Letters of Chemistry, Physics and Astronomy 4 (2014) 29-36.

[9] Z. S. Hamidi, N. N. M. Shariff, International Letters of Chemistry, Physics and Astronomy 5 (2014) 32-42.

[10] Z. S. Hamidi, N. N. M. Shariff, International Letters of Chemistry, Physics and Astronomy 5 (2014) 43-49. 